HERIOT

原国 WATT

UNIVERSITY

Heriot-Watt University

Research Gateway

\title{
Partial discharge detection using software defined radio
}

\section{Citation for published version:}

Mohamed, H, Lazaridis, P, Upton, D, Khan, U, Saeed, B, Jaber, A, Zhang, Y, Mather, P, Vieira, MFQ, Barlee, K, Atkinson, DSW \& Glover, IA 2017, Partial discharge detection using software defined radio. in 2016 International Conference for Students on Applied Engineering (ICSAE)., 7810220, IEEE. https://doi.org/10.1109/ICSAE.2016.7810220

\section{Digital Object Identifier (DOI): \\ 10.1109/ICSAE.2016.7810220}

\section{Link:}

Link to publication record in Heriot-Watt Research Portal

\section{Document Version:}

Peer reviewed version

\section{Published In:}

2016 International Conference for Students on Applied Engineering (ICSAE)

\section{Publisher Rights Statement:}

(C) 2019 IEEE. Personal use of this material is permitted. Permission from IEEE must be obtained for all other uses, in any current or future media, including reprinting/republishing this material for advertising or promotional purposes, creating new collective works, for resale or redistribution to servers or lists, or reuse of any copyrighted component of this work in other works.

\section{General rights}

Copyright for the publications made accessible via Heriot-Watt Research Portal is retained by the author(s) and / or other copyright owners and it is a condition of accessing these publications that users recognise and abide by the legal requirements associated with these rights.

\section{Take down policy}

Heriot-Watt University has made every reasonable effort to ensure that the content in Heriot-Watt Research Portal complies with UK legislation. If you believe that the public display of this file breaches copyright please contact open.access@hw.ac.uk providing details, and we will remove access to the work immediately and investigate your claim. 


\title{
Partial Discharge Detection Using Software Defined Radio
}

\author{
H. Mohamed ${ }^{1}$, P. Lazaridis ${ }^{1}$, D. Upton ${ }^{1}$, U. Khan ${ }^{1}$, B. Saeed ${ }^{1}$, A. Jaber ${ }^{1}$, Y. Zhang ${ }^{1}$, P. Mather ${ }^{1}$, M. F. Q. Vieira ${ }^{2}$, K. \\ Barlee $^{3}$, D. S. W. Atkinson ${ }^{3}$, and, I. A. Glover ${ }^{1}$ \\ ${ }^{1}$ Department of Engineering \& Technology, University of Huddersfield, Huddersfield HD1 3DH, UK \\ ${ }^{2}$ Department of Electrical Engineering, Universidade Federal de Campina Grande, Campina Grande, \\ Brazil
}

${ }^{3}$ Department of Electronic and Electrical Engineering, University of Strathclyde, Glasgow G1 1XW

E-mail: Hamd.Mohamed@hud.ac.uk

\begin{abstract}
Partial discharge (PD) is an electrical discharge that occurs within part of the dielectric separating two HV (High Voltage) conductors. PD causes damage to the dielectric which typically deteriorates with time. If left untreated, PD may result in catastrophic insulation failure, destruction of HV equipment, and disruption of power supply. The emergence of wireless network technology and software defined radio has opened new opportunities in PD monitoring and early detection of failures. This paper proposes the use of Universal Software Radio Peripheral (USRP) technology for PD detection.
\end{abstract}

Keywords-Partial discharge detection; Spectrum sensing; USRP; Software Defined Radio.

\section{INTRODUCTION}

Electrical insulation failure is an important cause of unplanned outage of power systems. Internal Partial Discharge (PD) in electrical systems indicates inclusions of material with dielectric properties distinct from the surrounding insulation. This foreign material may be gas filling a void in a solid or liquid dielectric. When two materials with different permittivity are subject to a voltage, the electric field is greatest in the region of lower permittivity. Electrical breakdown can occur in this region without occurring elsewhere. Repeated partial discharge damages the insulation and may eventually result in complete discharge (i.e. flashover), [1]. Partial discharge measurement may be used to monitor the integrity of the insulating material. In particular PD radiations resulting from the short current pulses characteristic of the PD process can be detected by a network of radiometers and detect $\mathrm{PD}$, locate its origin and monitor its severity. Typically, such radiation has significant spectral density between about $100 \mathrm{MHz}$ and $1 \mathrm{GHz}$. Electrical transients, due to switching devices for example, represent a potential source of broadband radiation that may be confused with, or mask, PD. Narrowband communication signals originating from TV and radio broadcast transmitters can also cause significant interference. Such interference is generally distinguishable from PD, however, on the basis of its narrowband character. Its effect may also be mitigated in applications where the PD sensor network is restricted to a well-defined geographically area of modest size (such as an electrical substation) by the fact that such signals typically arise from a distant transmitter and hence 'biases' the radiometric measurements approximately equally for all the sensors in network. Spatial variation of measured PD is, therefore, likely to predominantly reflect signals (in particular PD radiation) of local origin, [2].

\section{RELATED WORK}

Several electromagnetic (EM) approaches have been employed for the detection of partial discharge in the context of monitoring the integrity of high voltage infrastructure. The majority of existing systems employ conventional approaches for partial discharge detection such as electric parameter analysis based schemes, chemical analysis, and other sensing node (sensor) based approaches. These methods involve the detection of radiated electromagnetism emitted due to PD activity using electromagnetic (EM) sensors which can provide valuable information on the condition of $\mathrm{HV}$ equipment. In existing literature, the EM radiation in isotropic media has been studied in depth. Bojovschi et al., [3], investigated the partial discharge detection using the radiation spectra along the transmission line. According to the electromagnetic radiation spectra of partial discharge, the propagation of EM radiation in high voltage insulating materials behaves similar to radiation from dipoles or multipoles. Generally, correct detection of partial discharge depends on the precise interpretation of the EM spectra emitted from discharge zones. Several researchers performed investigations on the coupling between the EM radiation, generated by $\mathrm{PD}$, and electrical or electronic detection systems and confirmed its great significance for $\mathrm{HV}$ power infrastructure in order to detect electrical faults.

Atkinson et al., [4], were the first to research partial discharge detection using a wireless sensor network (WSN). It was a network possessing $\mathrm{PD}$ radiometer sensors, individually interfaced to a WSN node. The previously mentioned researchers discovered that there are two EM compatibility issues for WSN based PD detection that need to be taken into consideration: the increase of the measured radiometric PD power by the WSN signals might cause an overestimation of 
the partial discharge intensity, and the possible interference caused by communications signals, such as radio and television transmissions, received by the WSN, may cause difficulties in receiving $\mathrm{PD}$ events. The predominant approaches for alleviating such issues are the selection of a certain optimal frequency band and WSN radio operating band. In general, most of the PD energy occupies the frequency band of 50-800 MHz, while the frequency band for the PD wireless sensor network WSN is the $2.45 \mathrm{GHz}$ ISM band. Previous studies also suggest that there is no significantly adverse impact on the wireless sensor network performance for partial discharge detection caused by the substation electromagnetic environment. The optimal signal filtering at the front-end of the PD radiometer may reduce the influence of the presence of interfering communication signals on the PD intensity estimation, [5].

Zhang et al., [6], proposed the received signal strength, using WSN, as a metric to monitor and locate PD signals in HV electrical substations, stating the advantage of using an RRS (received signal strength) approach does not require accurate time synchronization between nodes nor the use of an antenna array. Suryandi et al., [7], demonstrated identification of single and multiple PD sources in high power transformers using UHF enriched signal sensors with de-noising tools. Chang et al., [8], measured PD in GIS using commercial inductive sensors and the current signals were classified using an Artificial Neural Network (ANN). Similar work has been done in [9], where authors focused on employing a bow-tie antenna as a UHF sensor to achieve a $300 \mathrm{MHz}$ reception bandwidth. Researchers in [10] presented a novel ultra wideband (UWB) printed monopole for PD WSN detection. Huang et al., [11], employed SDR for spectrum sensing that explored the white spectrum in the TV band $(100-700 \mathrm{MHz})$ and used a chi-square test with cyclostationary features for spectral sensing.

A number of studies have been performed so far for effective partial discharge detection, however, not much effort has been placed on developing certain cost effective or medium cost PD detection schemes utilizing spectral discrimination, such as one using Software Defined Radio solutions. Recently, researchers from the University of Strathclyde have showed in their work how SDR devices can be used to perform automated sweeps of the RF spectrum, [12]. Furthermore, a wideband sensing device called USRP has been developed that possesses better sensing capabilities at higher frequencies and hence it can be a potential candidate for partial discharge detection. The use of USRP SDR can enable low cost and accurate PD detection, [13].

\section{HARDWARE/SOFTWARE OVERVIEW}

The SDR is a radio system in which some, or all, functions of the physical layer are defined and implemented in software instead of hardware, [13]. This means that these functions are programmable, which allows users to change the parameters (e.g. center frequency, bandwidth, and modulation) as well. The device chosen for the work reported here is shown in Figure 1.

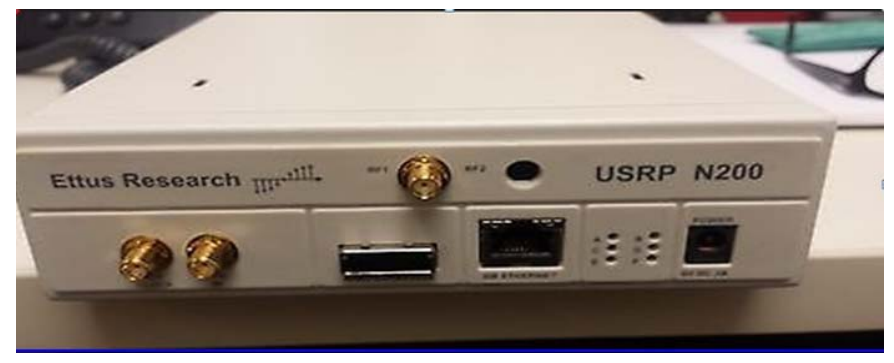

Fig. 1. USRP N200 Transceiver.

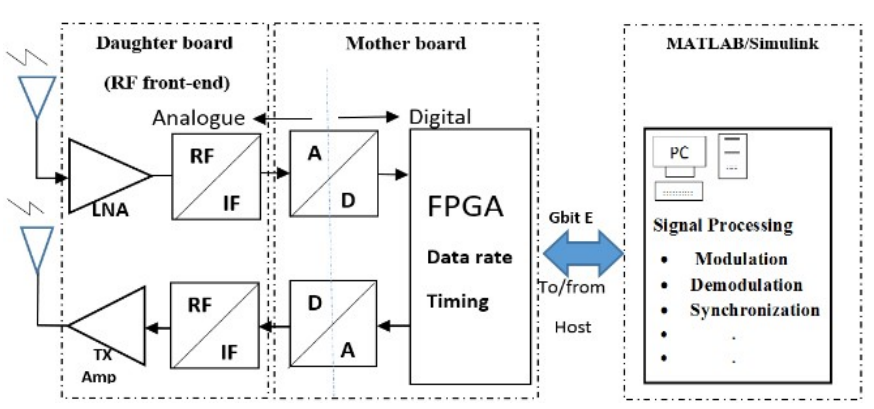

Fig. 2. USRP Block diagram.

Figure 2 is a block diagram of the USRP device. This SDR device uses an FPGA rather than an ASIC. The RF daughter board carries amplification, filtering and down conversion in the receive branch. The motherboard converts the received complex baseband signal from analogue to digital form. The FPGA implements the baseband signal processing (e.g. demodulation and synchronization) before transferring the processed signal to a host computer via an Ethernet cable.

\section{THE EXPERIMENTAL SET UP}

Figure 3 shows the experimental set up. It consists of a high voltage AC power supply, a PD emulator inside a wooden box, for health and safety reasons and a USRP N200 transceiver connected to a laptop via a Giga Ethernet cable. The USRP receives the PD signal through a wideband biconical antenna, and the received signal is processed in the USRP receiver and in a laptop using MATLAB code.

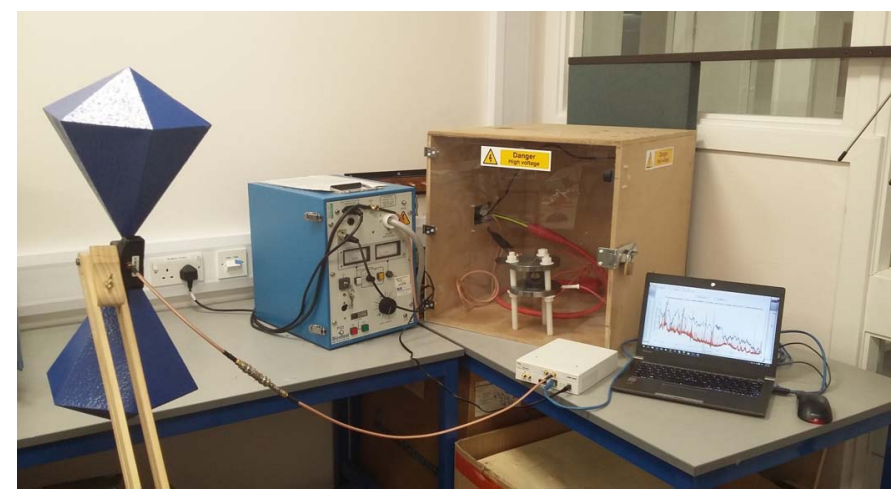

Fig. 3. Experimental setup. 
Some of the specifications of the USRP transceiver are shown in Table 1.

Table 1. Specifications of the USRP N200.

\begin{tabular}{|c|c|}
\hline Feature & Value \\
\hline $\begin{array}{c}\text { Sampling frequency of } \\
\text { ADC }\end{array}$ & $100 \mathrm{M}$ Samples $/ \mathrm{sec}$ \\
\hline ADC Resolution & $14 \mathrm{Bits}$ \\
\hline Frequency Accuracy & $2.5 \mathrm{ppm}$ \\
\hline Receiver Noise Figure & $5 \mathrm{~dB}$ \\
\hline Dimensions $(1 \mathrm{x} \mathrm{w} \mathrm{x}$ ) & $22 \times 16 \times 5 \mathrm{~cm}$ \\
\hline Weight & $1.2 \mathrm{~kg}$ \\
\hline Frequency range & $50-2200 \mathrm{MHz}$ \\
\hline
\end{tabular}

The USRP N200 transceiver with WBX daughter board has been selected due to the PD band 50-800 $\mathrm{MHz}$ being adequately covered. A particularly convenient feature of the USRP is that it allows high speed streaming of up to $50 \mathrm{MS} / \mathrm{s}$ in both directions.

\section{THE EXPERIMENTAL RESULTS}

The proposed SDR system has been developed using the USRP N200 transceiver. The performance of the proposed SDR system has been compared with a high-end portable spectrum analyzer. Spectral sensing has been performed and analyzed at the PD band of $50-800 \mathrm{MHz}$.

Figure 4 shows the measured spectrum using the USRP N200. The red curve is the spectrum when the PD signal is present while the blue curve is the spectrum when the PD signal is absent. By inspecting the spectra (i.e. in the presence and in the absence of a PD signal) it is clear that the level differences are up to approximately $23 \mathrm{~dB}$ in some places of the $50-800 \mathrm{MHz}$ frequency band. To validate the USRP SDR results, a portable spectrum analyser has also been employed.

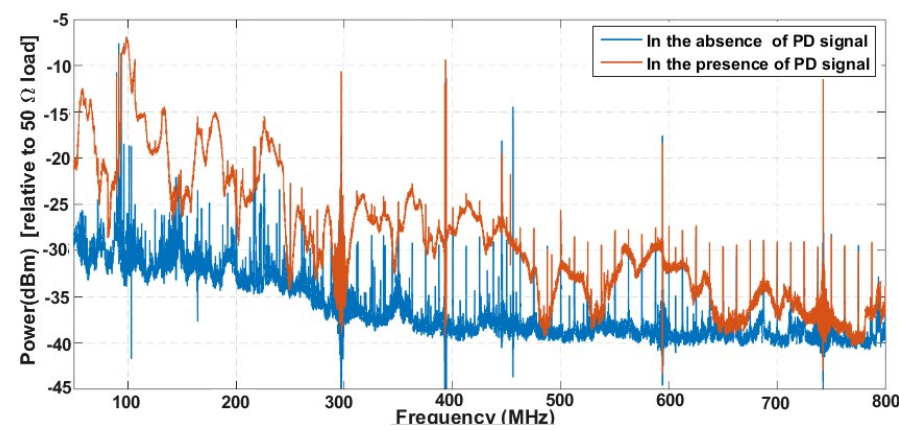

Fig. 4. PD signal measured spectrum using USRP N200 transceiver.

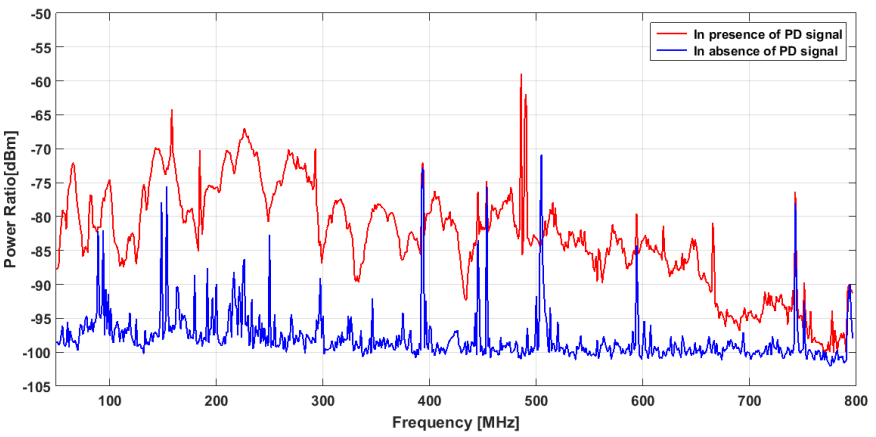

Fig. 5. PD signal measured spectrum using a spectrumanalyser.

Figure 5 shows the measured spectra in the absence and in the presence of a PD signal from the commercial spectrum analyser. The level differences here are up to $26 \mathrm{~dB}$. There is a great similarity between Figures. 4 and 5, although Figure 4 is noisier and not as accurate. This validates the results obtained using the USRP N200 Software Defined Radio solution for PD detection.

\section{CONCLUSION}

A PD detection method using SDR software by means of a USRP has been implemented. The performance of the selected spectrum sensing platform has been verified in experiments conducted using high-end commercial spectrum analysers. When comparing USRP results with spectrum analyser results, a good level of similarity is obtained. The results obtained in this research show that USRP can be an attractive low to medium cost solution for spectral PD detection.

\section{REFERENCES}

[1] Y. Zhang, J. M. Neto, D. Upton, A. Jaber, U. Khan, B. Saeed, P. Mather, P. Lazaridis, R. Atkinson, M.F. Q Vieira, I.A. Glover, "Radiometer monitoring system for partial discharge detection in substation," UK Festival of Radio Science, Manchester, Dec 2014.

[2] D. Upton, P. Mather, Y Zhang, A. Jaber, H. Ahmed, U Khan, et al, " Signal Conditioning Electronics for UHF Partial Discharge Detection and Location System, "UK Festival of Radio Science, Manchester, Dec 2014.

[3] A. Bojovschi, W. S. T. Rowe, and K. L. Wong "Radiation spectra of partial discharge in dielectrics, "IEEE Proceedings of the Australian Universities Power Engineering Conference, 1(6), Adelaide, Australia, Sep. 2009.

[4] Atkinson, K., and I. Glover, "A self-calibrating partial discharge W S N for condition monitoring in the future smart grid, "3rd IEEE PES ISGT Conference, Berlin, Oct. 2012.

[5] Q. Shan, I. Glover, A. Moore, P. Moore, I. Portugues, M. Judd, et al, " Impulsive noise environment of high voltage electricity transmission substations and its impact of the performance of ZigBee, " in Proc. Int. Conf. Wireless Commun. Mobile Comput, pp. 859-863, August 2008.

[6] Y. Zhang, D. Upton, A. Jaber, H. Ahmed, B. Saeed, P. Lazaridis, et al, " Radiometric wireless sensor network monitoring of partial discharge sources in electrical substations ". Hindawi International Journal of Distributed Sensor Networks, 179, August 2015.

[7] A. Suryandi and U. Khayam, "Design of new shape of bowtie antenna with edge modification for UHF partial discharge measurement," Power Engineering and Renewable Energy (ICPERE), International Conference, pp. 257-261, Bali, Indonesia, Dec. 2014.

[8] H. Sinaga, B. Phung, T Blackburn, "Recognition of single and multiple partial discharge sources in transformers based on ultra-high frequency 
Signals," Generation, Transmission and Distribution, IET, vol. 8, no. 1, pp. 160-169, Jan. 2014.

[9] H. Chang, F. Gu, H. Chen and C. Kuo, "Partial discharge measurement and pattern recognition in gas insulated switchgear," Lightning APL, 7th Asia-Pacific International Conference, pp.459-462, Chengdu, China Nov. 2011.

[10] Y. Zhang, D. Upton, A. Jaber, U. Khan, B. Saeed, H. Ahmed, et al, " An ultrawideband patch antenna for UHF detection of partial discharge, " In Radio Science Conference (URSI AT-RASC), 2015 1st URSI Atlantic , pp. 1-1, Gran Canaria, Spain, May 2015.

[11] L. Huang, Q. Deng, X. Qiao, D. Gu, W. Wang, H. Yang, "SDR implementation of cognitive radio: Sensing and disjoint spectrum access, "Communications and Networking in China, China, COM2009, Fourth International Conference, pp. 1-5, Aug. 2009.

[12] R. Stewart, K. Barlee, D. Atkinson, and L. Crockett, " Software Defined Radio using MATLAB \& Simulink and the RTL-SDR" 1st ed., Strathclyde Academic media, October 2015.

[13] H. Mohamed, P. Lazaridis, D. Upton, U. Khan, B. Saeed, K. Barlee et al, "Partial discharge detection using low cost RTL-SDR model for wideband spectrum sensing, " ICT 2016, Thessaloniki, Greece, May 2016. 